\title{
Analysis of Gabor Parameters for Handwritten Numeral Recognition by Experimental Design
}

\author{
Shunji Uchimura, Kiyoshi Mizuno, Yoshihiko Hamamoto and Shingo Tomita
}

Faculty of Engineering, Yamaguchi University, Ube, 755-8611, Japan

\begin{abstract}
Gabor filter-based features are useful for handprinted character recognition. One needs to optimize Gabor filter parameters because the performance of Gabor features depends strongly on Gabor filter parameters. One way to find the optimal values of the parameters is to analyze statistically the influence of the parameters on the error rate. In this paper, we discuss a statistical analysis of Gabor parameters for handwritten numeral recognition by experimental design. Our statistical analysis shows that optimal values of standard deviations $\sigma_{x}$ and $\sigma_{y}$ in Gabor filter are functions of the wavelength of the filter. In addition, it is shown that optimal values of $\sigma_{x}$ and $\sigma_{y}$ can be separately set on the condition that $\sigma_{x} \geq \sigma_{y}$.
\end{abstract}

\section{Introduction}

In the handprinted character recognition field, significant research efforts have been made. However, no machine with a performance level similar to that of humans is developed. It is well known that the performance of a character recognition system depends strongly on the features used. Hence, we believe that by using the features similar to those extracted by the human visual system, the machine can achieve the performance similar to that of humans. Gabor filters[1] have been shown to be good fits to the receptive field profiles of simple cells in the striate cortex[2],[3]. This characteristic suggests that the Gabor filter-based features seem to be similar to features extracted by the humans and thus may be effective in classifying characters. Therefore, a Gabor filter-based method for handprinted character recognition is proposed [4], and it is shown that the Gabor filter-based features are very effective in classifying both handwritten numeral character [4] and handprinted Chinese character[5].

It is known that the classification performance of Gabor filter-based features depends strongly on Gabor filter parameters such as wavelength $\lambda$ and two-dimensional spread $\sigma_{x}$ and $\sigma_{y}$ of the filter [4]. Thus, one needs to determine optimal values of filter parameters. In some applications of the Gabor filter, values of $\sigma_{x}$ and $\sigma_{y}$ are determined on some conditions. For example, values of $\sigma_{x}$ and $\sigma_{y}$ are equal and constant in $[6],[7]$. On the other hand, some authors $[8],[9]$ point out that $\sigma_{x}$ and $\sigma_{y}$ are a function of $\lambda$, respectively. For handprinted character recognition, however, very little is known about the conditions of optimal values of $\sigma_{x}$ and $\sigma_{y}$. We expect that if we find the conditions of optimal values 
of $\sigma_{x}$ and $\sigma_{y}$ for handprinted character recognition, the conditions are useful to optimize the parameters which minimize the error rate.

The purpose of this study is to study optimization of $\sigma_{x}$ and $\sigma_{y}$ for handwritten numeral recognition. In order to find the conditions, we statistically analyze the effects of Gabor filter parameters on the error rate by experimental design[10]; this is a useful tool that provides a strategy of experimentation and a statistical method for analyzing effects of factors on observations. Our statistical analysis shows that the optimal values of $\sigma_{x}$ and $\sigma_{y}$ are respectively functions of $\lambda$. In addition, it is also shown that the optimal values can be separately set on the condition that $\sigma_{x} \geq \sigma_{y}$.

\section{Gabor Features}

A two-dimensional Gabor filter is defined by

$$
f\left(x, y, \theta_{k}, \lambda, \sigma_{x}, \sigma_{y}\right)=\exp \left[-\frac{1}{2}\left\{\frac{R_{1}^{2}}{\sigma_{x}^{2}}+\frac{R_{2}^{2}}{\sigma_{y}^{2}}\right\}\right] \cdot \exp \left\{i \frac{2 \pi R_{1}}{\lambda}\right\}
$$

where $R_{1}=x \cos \theta_{k}+y \sin \theta_{k}$ and $R_{2}=-x \sin \theta_{k}+y \cos \theta_{k} . \lambda$ and $\theta_{k}$ are the wavelength and orientation of the sinusoidal plane wave, respectively. $\sigma_{x}$ and $\sigma_{y}$ are the standard deviations of the Gaussian envelope along the $x$ - and $y$ directions, respectively. A rotation of the $x-y$ plane by an angle $\theta_{k}$ will result in a Gabor filter at orientation $\theta_{k}$. In this paper, the value of $\theta_{k}$ is given by $\theta_{k}=\pi(k-1) / m, \quad k=1,2, \cdots, m$, where $m$ denotes the number of orientations.

The Gabor feature can be viewed as the response of the Gabor filter located at a sampling point. The response is obtained by convolving the filter with an image. Gabor filters extract the orientation-dependent edge-like features from as small an area as possible. The sampling points were set according to the following procedure[4]: (1) find the circumscribing frame of the character in an image, (2) extend the circumscribing frame by $\alpha$ dots, (3) arrange 64 sampling points in the extended frame at regular intervals. From preliminary experiments[4], we used $\alpha=3$. First, we select values of the filter parameters $\lambda, \sigma_{x}$ and $\sigma_{y}$, and let their values be fixed. Then, the Gabor feature corresponding the filter with orientation $\theta_{k}$ at a sampling point $(X, Y)$ is defined as:

$$
g\left(X, Y, \theta_{k}, \lambda, \sigma_{x}, \sigma_{y}\right)=\left|\sum_{x=-X}^{N-X-1} \sum_{y=-Y}^{N-Y-1} I(X+x, Y+y) f(\cdot)\right|
$$

where $I(x, y)$ denotes an $N \times N$ binary image and $|z|$ denotes the absolute value of a complex number $z$. For each sampling point, $m$ Gabor features can be obtained. Since 64 sampling points are used, $64 m$ Gabor features can be obtained for each image.

\section{Experimental Design for Analyzing Gabor Parameters}

We describe our application of experimental design[10] for analyzing Gabor parameters. 
It is known that the error rate of Gabor features depends strongly on Gabor filter parameters $\lambda, \sigma_{x}$ and $\sigma_{y}$. Therefore, we consider an experiment with three factors $\lambda, \sigma_{x}$ and $\sigma_{y}$. From preliminary experiments[4], we use the following levels of the three factors.

$$
\lambda: 3 \sqrt{2}, 4 \sqrt{2}, 5 \sqrt{2}, 6 \sqrt{2}, 7 \sqrt{2} \quad \sigma_{x}: 2,3,4,5 \quad \sigma_{y}: 2,3,4,5 .
$$

Thus, $80(=5 \times 4 \times 4)$ runs are made in an experimental block. In order to obtain more precise estimates of the effects, the runs are made in each of 10 experimental blocks. That is, we treat a randomized complete block design with three factors.

To analyze the effects of factors, we assume a linear statistical model[10] as that of the error rate. Further, analysis of variance is conducted to know whether the main or interaction effects are significant. In addition, to know whether two mean error rates differ significantly, the difference of the two mean error rates is tested by using $t$ test.

\section{Experimental Results}

We used the ETL-1 database[11] (10 classes, 1400 images per class) to evaluate the error rate of the Gabor features. Binarization was applied on the images. After all, each sample is described as a $64 \times 64$ binary image. The value of $m$ was set to 4 , thus the number of Gabor features for an image was 256 . We used the holdout method[12] for error estimation and adopted a nearest neighbor(1NN) classifier. The following experiment was done.

1. Replicate the following (a) and (b) 10 times.

(a) Divide randomly the 1400 available samples into 400 training and 1000 test samples for each class.

(b) For all the set of levels of $\lambda, \sigma_{x}$ and $\sigma_{y}$,

i. Obtain the Gabor features of all the available samples.

ii. Design a 1-NN classifier by using the training samples.

iii. Test the classifier by using the test samples to compute the error rate.

2. Analyze the variance of the obtained error rates. Test the significance of difference between the minimum mean of error rate and the other means.

The analysis of variance is shown in Table 1 . Figs. 1 and 2 plot the mean error rates against factors $\sigma_{x}$ and $\sigma_{y}$ for each level of factor $\lambda$, respectively. Fig. 3 plots the mean error rates against factor $\sigma_{x}$ for each level of factor $\sigma_{y}$.

From Table 1, Figs.1 and 2, we see that the interaction effects of two factors $\left(\lambda \times \sigma_{x}, \lambda \times \sigma_{y}\right)$ are significant. This means that the optimal values of $\sigma_{x}$ and $\sigma_{y}$ must be determined for each value of $\lambda$, thus, the optimal values of $\sigma_{x}$ and $\sigma_{y}$ are functions of $\lambda$. This fact agrees with those presented in [8],[9]. On the other hand, Table 1 shows that the interaction effect of $\sigma_{x} \times \sigma_{y}$ is smaller than the other two factor interactions. Moreover, in Fig.3, we see that the lines of $\sigma_{y}$ are 
approximately parallel, indicating a lack of the interaction between $\sigma_{x}$ and $\sigma_{y}$. These mean that the optimal values of $\sigma_{x}$ and $\sigma_{y}$ can be set separately.

Table 2 shows the optimal values of $\left(\lambda, \sigma_{x}, \sigma_{y}\right)$ and the error rates which don't significantly differ from the minimum mean error rate. From Table 2, we find that the relation of the optimal values between $\sigma_{x}$ and $\sigma_{y}$ is $\sigma_{x} \geq \sigma_{y}$. This relation is very interesting as compared with those used in $[6],[7]$.

Table 1. The analysis of variance. '**' denotes that the effect is significant at $1 \%$ level of significance.

\begin{tabular}{l|rrrr}
\hline $\begin{array}{l}\text { Source of } \\
\text { Variation }\end{array}$ & $\begin{array}{r}\text { Sum of } \\
\text { Squares }\end{array}$ & $\begin{array}{c}\text { Degrees of } \\
\text { Freedom }\end{array}$ & $\begin{array}{c}\text { Mean } \\
\text { Square }\end{array}$ & $F$ ratio \\
\hline$\lambda$ & 252.72 & 4 & 63.18 & $4720.60^{* *}$ \\
$\sigma_{x}$ & 89.52 & 3 & 29.84 & $2229.61^{* *}$ \\
$\sigma_{y}$ & 50.02 & 3 & 16.67 & $1245.72^{* *}$ \\
$\lambda \times \sigma_{x}$ & 221.35 & 12 & 18.45 & $1378.21^{* *}$ \\
$\lambda \times \sigma_{y}$ & 96.69 & 12 & 8.06 & $602.00^{* *}$ \\
$\sigma_{x} \times \sigma_{y}$ & 14.23 & 9 & 1.58 & $118.16^{* *}$ \\
$\lambda \times \sigma_{x} \times \sigma_{y}$ & 11.18 & 36 & 0.31 & $23.21^{* *}$ \\
Blocks & 6.78 & 9 & 0.75 & $56.3^{* *}$ \\
Error & 9.52 & 711 & 0.01 & \\
Total & 752.01 & 799 & & \\
\hline
\end{tabular}

Table 2. The optimal values of $\left(\lambda, \sigma_{x}, \sigma_{y}\right)$ and the error rates which don't differ significantly from the minimum mean error rate at $1 \%$ level of significance.

\begin{tabular}{ccc|c}
\hline$\lambda$ & $\sigma_{x}$ & $\sigma_{y}$ & Mean Error \\
& & Rate(\%) \\
\hline $4 \sqrt{2}$ & 3 & 2 & 2.95 \\
$5 \sqrt{2}$ & 3 & 3 & 3.04 \\
$4 \sqrt{2}$ & 3 & 3 & 3.07 \\
$4 \sqrt{2}$ & 4 & 2 & 3.08 \\
\hline
\end{tabular}

\section{Conclusions}

In this paper, we discussed a statistical analysis of Gabor parameters for handwritten numeral recognition by experimental design. From our statistical analysis, it was shown that optimal values of $\sigma_{x}$ and $\sigma_{y}$ are functions of $\lambda$. In addition, it was shown that the optimal values can be separately set on the condition that $\sigma_{x} \geq \sigma_{\mho}$. 


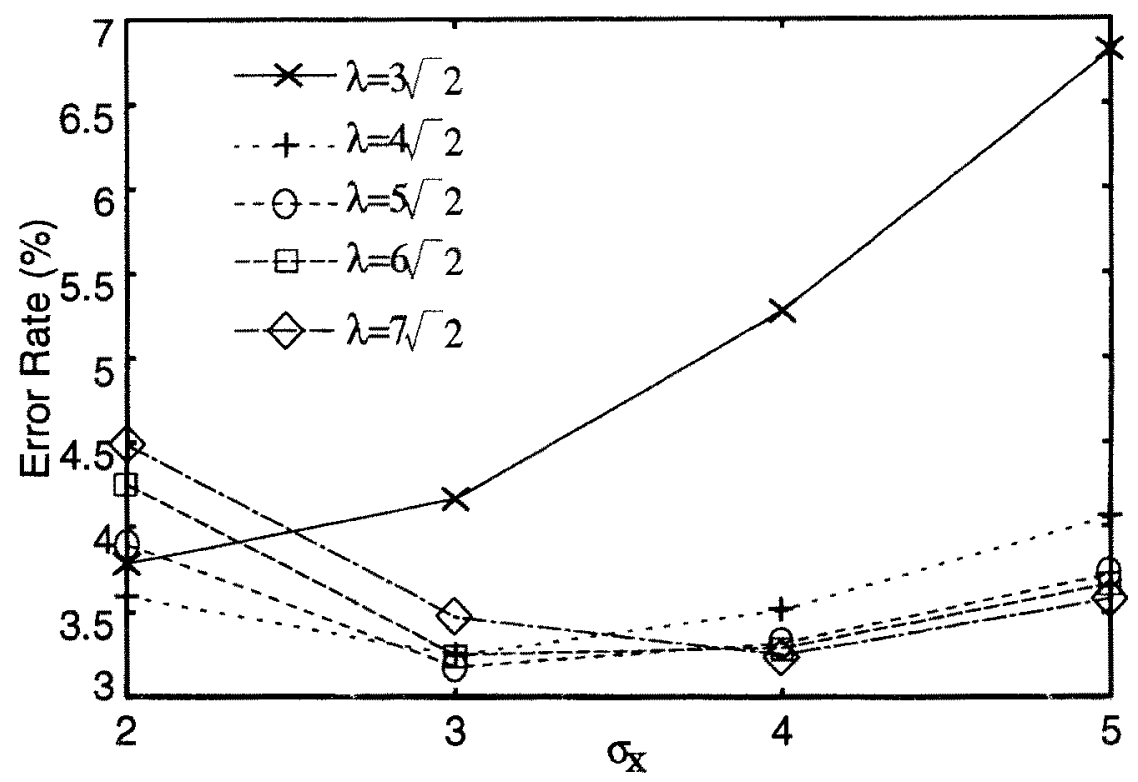

Fig. 1. The mean error rates against $\sigma_{x}$ for each level of $\lambda$.

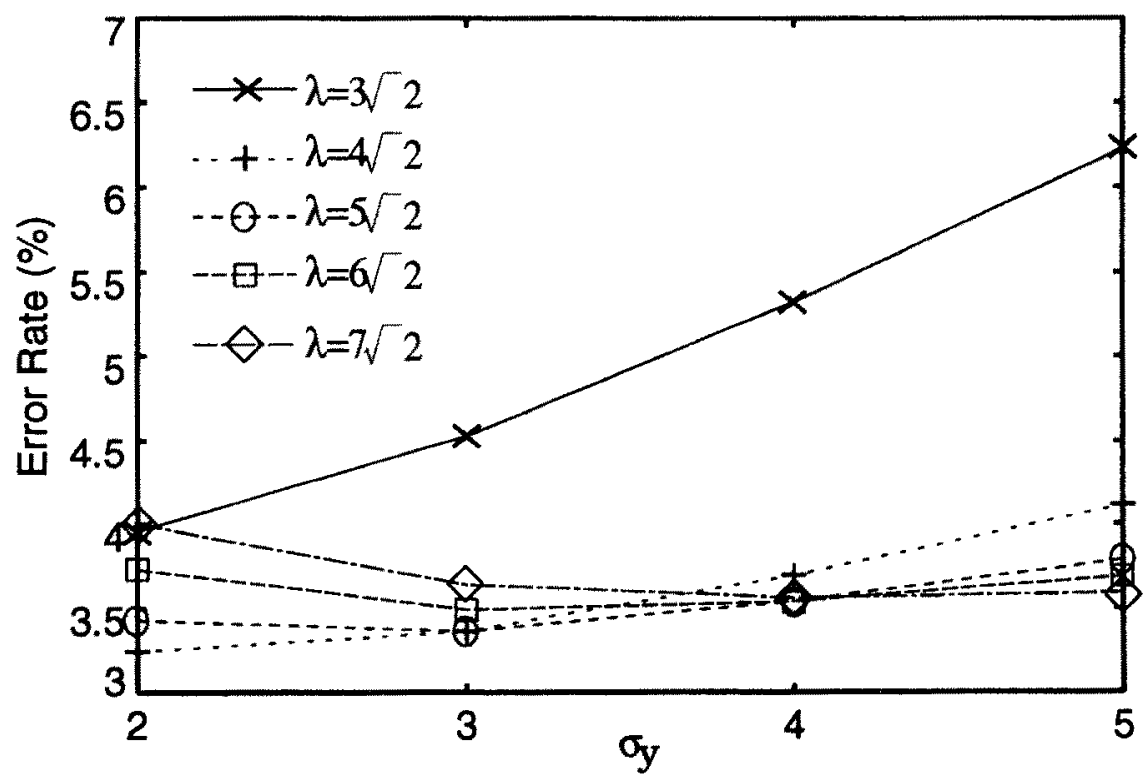

Fig. 2. The mean error rates against $\sigma_{y}$ for each level of $\lambda$. 


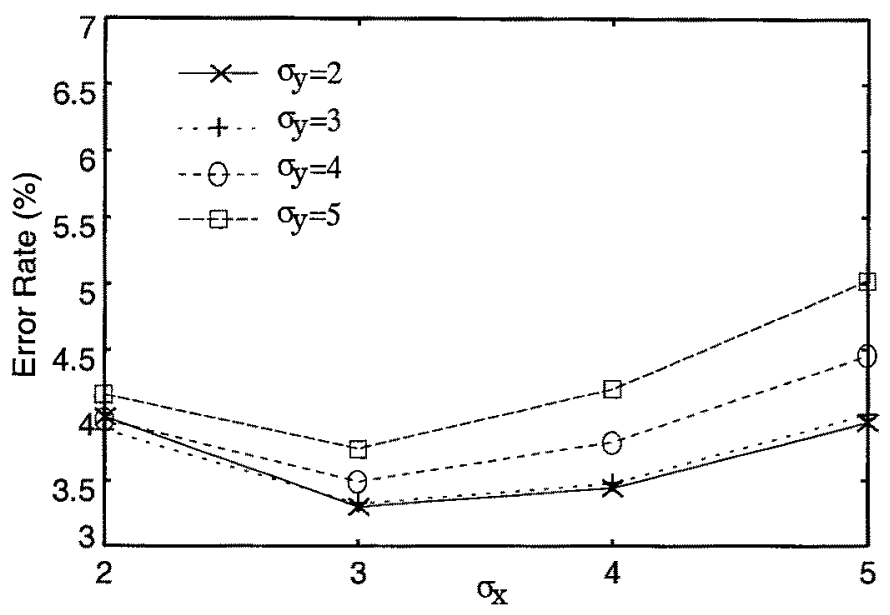

Fig. 3. The mean error rates against $\sigma_{x}$ for each level of $\sigma_{y}$.

\section{Acknowledgment}

We would like to thank the Electrotechnical Laboratory in Japan for providing the ETL-1 database.

\section{References}

1. Gabor, D.: Theory of communication. J. Inst. Elect. Engr. 93 (1946) 429-457

2. Daugman, J.G.: Two-dimensional spectral analysis of cortical receptive field profiles. Vision Res. 20 (1980) $847-856$

3. Marčelja, S.: Mathematical description of the responses of simple cortical cells. J. Opt. Soc. Am. 70 (1980) 1297-1300

4. Hamamoto, Y., et al: A Gabor filter-based method for recognizing handwritten numerals. Pattern Recognition 31 (1998) 395-400

5. Hamamoto, Y., et al: Recognition of handprinted Chinese characters using Gabor features. Proc. 3rd Int. Conf. Document Analysis and Recognition (1995) 819-823

6. Turner, M.R.: Texture discrimination by Gabor functions. Biol. Cybern. 55 (1986) $71-82$

7. Lampinen, J., Oja, E.: Distortion tolerant pattern recognition based on selforganizing feature extraction. IEEE Trans. Neural Networks 6 (1995) 539-547

8. Yamada, K., Tsukumo, J.: Consideration on stability of Gabor feature extraction and character recognition application. Tech. Rep. IEICE Japan PRU92-112 (1993) 75-82 (in Japanese)

9. Jain, A.K., Bhattacharjee, S.K.: Address block location on envelopes using Gabor filters. Pattern Recognition 25 (1992) 1459-1477

10. Montgomery, D.C.: Design and Analysis of Experiment, 4th Edition. John Wiley and Sons (1997) 
11. Yamamoto, K.: Present state of recognition method on consideration of neighbor points and its ability in common database. IEICE Trans. Information and Systems E79-D (1996) 417-422

12. Fukunaga, K.: Introduction to Statistical Pattern Recognition, 2nd Edition. Academic Press (1990) 